\title{
Functional and effective reorganization of the aging brain during unimanual and bimanual hand movements
}

\author{
Sara Larivière ${ }^{1,2}$ ( ) Alba Xifra-Porxas ${ }^{3}$ | Michalis Kassinopoulos ${ }^{3}$ | Guiomar Niso ${ }^{1,4,5}$ | \\ Sylvain Baillet ${ }^{1,2}$ | Georgios D. Mitsis ${ }^{6}$ | Marie-Hélène Boudrias ${ }^{7,8}$
}

${ }^{1} \mathrm{McC}$ Connell Brain Imaging Centre, Montreal Neurological Institute and Hospital, McGill University, Montréal, Québec, Canada

${ }^{2}$ Department of Neurology and Neurosurgery, McGill University, Montréal, Québec, Canada

${ }^{3}$ Department of Biological and Biomedical Engineering, McGill University, Montréal, Québec, Canada

${ }^{4}$ Center for Biomedical Technology, Technical University of Madrid, Madrid, Spain

${ }^{5}$ Biomedical Image Technologies, Technical University of Madrid and CIBER-BBN, Madrid, Spain

${ }^{6}$ Department of Bioengineering, McGill University, Montréal, Québec, Canada

${ }^{7}$ School of Physical and Occupational Therapy, McGill University, Montréal, Québec, Canada

${ }^{8}$ Center for Interdisciplinary Research in Rehabilitation of Greater Montreal (CRIR), Montréal, Québec, Canada

Correspondence

Marie-Hélène Boudrias, PhD, School of Physical and Occupational Therapy, Hosmer House, Room H-206, McGill University, 3654 Prom Sir-William-Osler, Montréal, Québec H3G 1Y5, Canada.

Email: mh.boudrias@mcgill.ca

Funding information

Canada Foundation for Innovation, Grant/ Award Numbers: 34277, 34362; Fondation Brain Canada, Grant/Award Number: PSG15-3755; Fonds de Recherche du Québec Nature et Technologies, Grant/Award Number: 2016-PR191780; Foundation for the National Institutes of Health, Grant/Award Number: 2R01EB009048-05; Natural Sciences and Engineering Research Council of Canada, Grant/Award Number: 436355-13

\begin{abstract}
Motor performance decline observed during aging is linked to changes in brain structure and function, however, the precise neural reorganization associated with these changes remains largely unknown. We investigated the neurophysiological correlates of this reorganization by quantifying functional and effective brain network connectivity in elderly individuals ( $n=11$; mean age $=67.5$ years), compared to young adults ( $n=12$; mean age $=23.7$ years), while they performed visuallyguided unimanual and bimanual handgrips inside the magnetoencephalography (MEG) scanner. Through a combination of principal component analysis and Granger causality, we observed agerelated increases in functional and effective connectivity in whole-brain, task-related motor networks. Specifically, elderly individuals demonstrated (i) greater information flow from contralateral parietal and ipsilateral secondary motor regions to the left primary motor cortex during the unimanual task and (ii) decreased interhemispheric temporo-frontal communication during the bimanual task. Maintenance of motor performance and task accuracy in elderly was achieved by hyperactivation of the task-specific motor networks, reflecting a possible mechanism by which the aging brain recruits additional resources to counteract known myelo- and cytoarchitectural changes. Furthermore, resting-state sessions acquired before and after each motor task revealed that both older and younger adults maintain the capacity to adapt to task demands via network-wide increases in functional connectivity. Collectively, our study consolidates functional connectivity and directionality of information flow in systems-level cortical networks during aging and furthers our understanding of neuronal flexibility in motor processes.
\end{abstract}

\section{KEYWORDS}

aging, granger causality, magnetoencephalography, motor control, network connectivity

\section{1 | INTRODUCTION}

Advancing age is associated with decreased cognitive and motor performance in humans (Enoka et al., 2003; Seidler et al., 2010). Motor decline often affects skills that are necessary to perform many daily life tasks as, for instance, hand motor control (e.g., reaching and grasping objects), bimanual coordination (e.g., tying shoelaces), as well as gait and balance (e.g., walking; Maes, Gooijers, de Xivry, Swinnen, \& Boisgontier, 2017; Seidler et al., 2010). With an ever-increasing aging society (Krueger et al., 2015), identifying the neural correlates underpinning the deterioration of motor control has become a primary focus of research (Song et al., 2014; Tomasi \& Volkow, 2012). Early studies highlighted age-related structural atrophy and aberrant functional activity in primary and secondary motor areas (Calautti, Serrati, \& Baron, 2001; Mattay et al., 2002; Sullivan, 
Rohlfing, \& Pfefferbaum, 2010; Ward, Swayne, \& Newton, 2008), while more recent accounts have selectively focused on altered communication in functional brain networks involved in sensory and cognitive functions (Fujiyama et al., 2016; King et al., 2017; Park, Boudrias, Rossiter, \& Ward, 2012; Siman-Tov et al., 2016). Despite these quantitative reports, a neurobiological framework consolidating whole-brain functional connectivity and directionality of information flow within macroscale cortical networks recruited during unimanual and bimanual movements remains to be established in healthy aging.

Brain reorganization in aging is believed to take place in order to maintain motor performance despite gray and white matter volume loss (Mora, Segovia, \& del Arco, 2008; Sullivan et al., 2010). Age-related thinning of the cerebral cortex occurs predominantly in the primary motor cortex (M1) and calcarine sulcus (Salat et al., 2004). In addition to structural changes, growing evidence suggests a compromised functioning of the motor network during healthy aging (for comprehensive reviews, see Maes et al., 2017; Seidler et al., 2010). One of the main theories that has emerged to explain brain reorganization in light of these age-related structural changes posits that activity levels within a given motor-related area is increased in an attempt to compensate for neuronal loss and myelin deterioration (Cabeza, Anderson, Locantore, \& Mclntosh, 2002). Magnetoencephalography (MEG) represents a powerful tool to investigate task-related and resting-state dynamic cortical networks with millisecond resolution and consequently unravel the complex functional changes involved in healthy aging. Notably, MEG captures real-time neuronal activity by measuring extracranial neuromagnetic fields and is less prone to spatial distortions caused by head tissues than electroencephalography (Baillet, 2017; Supek \& Aine, 2014). An increasing number of MEG studies have shown great promise for mapping the brain's spatiotemporal characteristics in the context of aging. For instance, resting-state MEG studies have reported reduced slow oscillatory activity $(<8 \mathrm{~Hz})$ but an increase in faster oscillations (8-30 Hz) in older adults (Bruce, Bruce, \& Vennelaganti, 2009; Kielar et al., 2016; Vlahou, Thurm, Kolassa, \& Schlee, 2014). These findings are in line with a previous study reporting faster neuronal firing rates and oscillation frequencies in task-activated cortical regions in elderly individuals (Hong \& Rebec, 2012). This effect is thought to indicate the presence of a compensatory mechanism that counteracts the decrease in nerve conduction velocity due to white matter atrophy in healthy aging (Kielar et al., 2016). While the examination of brain oscillations can provide direct information on networks electrophysiology, the analysis of connectivity time series using this modality remains in its infancy, with few available methods (O'Neill et al., 2017).

Harnessing the high spatiotemporal resolution of MEG, our study introduces a novel method to track age-related functional and effective connectivity changes in whole-brain networks underlying the production of unimanual and bimanual handgrips. Our main hypothesis was that the aging brain counteracts neurobiological changes by recruiting additional resources in motor-related networks (i.e., increased functional connectivity or hyperactivity). Consistent with the extensive literature on the role of stimulus-locked evoked alpha $(8-12 \mathrm{~Hz})$ and beta $(13-30 \mathrm{~Hz})$ responses during motor tasks (Crone et al., 1998; Willemse et al., 2010; Yuan et al., 2010), we employed principal component analysis (PCA) to derive brain networks that were (i) within those frequency ranges, (ii) consistent across all participants to allow direct comparison of network connectivity between groups, and (iii) specifically underlying hand movements. In line with previous evidence highlighting a loss of hemispheric asymmetry during normal aging (Heuninckx, Wenderoth, Debaere, Peeters, \& Swinnen, 2005; Heuninckx, Wenderoth, \& Swinnen, 2008; Ward \& Frackowiak, 2003), we further used Granger causality analysis to evaluate the directionally of information flow within the identified task-related networks. We expected that elderly individuals would show an increase in bilateral causal influences among motor-related regions. Aside from reorganization of task-based networks, modulation of restingstate connectivity following performance of motor tasks has been frequently demonstrated in fMRI studies of young adults (Ma et al., 2010; Tung et al., 2013), whereas evidence of task-induced modulation in older adults remains scarce (Solesio-Jofre et al., 2018). Leveraging an innovative study design with interspersed resting-state and task sessions, we were able to assess whether aging affects resting-state network connectivity immediately following the performance of unimanual and bimanual hand movements. We hypothesized that the expected increase in task-related connectivity in elderly subjects would lead to a subsequent increase in resting-state connectivity immediately following the task. This would provide robust evidence that performing a repetition of handgrip movements can result in age-specific functional changes in the brain, therefore providing insights into the mechanisms by which the healthy older brain adapts to different levels of task demands.

\section{2 | METHODS}

\section{1 | Participants}

MEG recordings as well as structural T1 $w$ images were collected from 12 healthy young adults (mean age $=23.7$ years) and 11 healthy elderly individuals (mean age $=67.4$ years). Details regarding data acquisition and preprocessing are described in the Supporting Information. Details regarding demographic information and behavioral performance are presented in Table 1. Both groups were matched with respect to gender and education. Inclusion criteria for all participants were as follows: (i) no present or previous history of a psychiatric conditions, (ii) aged between 18-30 years (young group) and 60-75 years (elderly group), and (iii) right-handed according to the Edinburgh Handedness Inventory (Oldfield, 1971). Exclusion criteria included: (i) contraindications for MRI, or other limitations that would interfere with MRI or MEG data acquisition (e.g., claustrophobia, metal implants) and (ii) a Mini-Mental State Examination (MMSE) score $\leq 24$. Written informed consent was obtained from all participants. The study was approved by the Research Ethics Board of the Montreal Neurological Institute and Hospital, McGill University.

\section{2 | Experimental design}

As detailed in Supporting Information, motor performance of both hands was assessed for each participant via measurements of (i) handgrip strength, (ii) fine manual dexterity (nine hole peg test; NHPT), and (iii) unilateral gross manual dexterity (box and block test; BBT). Motor performance scores for the dominant hand (right hand) and nondominant hand (left hand) were used for group comparison. 
TABLE 1 Participants' demographic information and behavioral scores

\begin{tabular}{|c|c|c|}
\hline Variable & Young & Elderly \\
\hline Sex (male/female) & $8 / 4$ & $8 / 3$ \\
\hline Handedness (right/left) & $12 / 0$ & $11 / 0$ \\
\hline Age (years) ${ }^{a}$ & $23.7(2.9)$ & $67.4(3.9)$ \\
\hline BBT (right) ${ }^{\mathrm{b}}$ & $67.5(5.5)$ & $57.1(4.2)$ \\
\hline BBT (left) ${ }^{b}$ & $66.7(5.5)$ & $56.8(4.8)$ \\
\hline NHPT (right) ${ }^{b}$ & $0.58(0.1)$ & $0.44(0.04)$ \\
\hline NHPT (left) ${ }^{c}$ & $0.52(0.1)$ & $0.41(0.06)$ \\
\hline Grip strength (right) & $46.2(15.1)$ & $39.1(9.3)$ \\
\hline Grip strength (left) & $44.1(16.1)$ & $34.8(7.9)$ \\
\hline
\end{tabular}

Note. Standard deviations are in parentheses. BBT: box and block test; NHPT: nine-hole peg test.

aElderly $>$ young, $p<0.0001$

${ }^{\text {b}}$ Young $>$ elderly, $p<0.0005$

${ }^{\mathrm{C}}$ Young $>$ elderly, $p<0.01$.

All participants underwent three separate 5 min resting-state sessions, interspersed with two isometric handgrip tasks (Figure 1). During each resting-state session, participants were instructed to keep their eyes open and fixate on a cross. The first motor task consisted of 50 unimanual, visually-paced, isometric right-handgrips, in which subjects had to apply force to track a ramp target. Prior to scanning, subjects were asked to grip the manipulandum with maximum force in order to assess their maximum voluntary contraction (MVC). These values were then used to set the subject-specific target forces of $15 \%$ and $30 \%$ of MVC. In each trial, participants had to maintain a steady force at $15 \%$ of MVC for $3 \mathrm{~s}$, followed by a linear increase of $3 \mathrm{~s}$ to reach and maintain a steady force at $30 \%$ of MVC for $3 \mathrm{~s}$. The second motor task consisted of 50 bimanual, visuallypaced, isometric handgrips performed at $15 \%$ of MVC ( 6 s each).

\section{3 | Task-based functional connectivity and statistical analyses}

The task-based functional connectivity analysis pipeline (Supporting Information Figure S1) was conducted in Brainstorm (Tadel, Baillet, Mosher, Pantazis, \& Leahy, 2011) and MATLAB, and was similar to that used in previous research (Larivière et al., 2017; Larivière, Ward, \& Boudrias, 2018; Whitman et al., 2016). Specifically, for every participant, the task-related data were down-sampled to $160 \mathrm{~Hz}$ and epoched offline with a poststimulus time window of $9,000 \mathrm{~ms}$ (unimanual task) and 6,000 ms (bimanual task) with the first time point (time $=0$ ) corresponding to stimulus onset. A linearly-constrained minimum variance (LCMV) beamformer spatial filtering approach (Van Veen \& Buckley, 1988) was used on the subject-specific task-averaged epoched data to reconstruct a single time series for each of the 148 cortical brain regions defined by the Destrieux sulcogyral-based atlas (Destrieux, Fischl, Dale, \& Halgren, 2010). For each pre-defined source location (i.e., brain region), activity was estimated at each vertex and subsequently averaged to produce a single time series per brain region. Time-frequency decomposition of source time series was then performed using Morlet wavelets (Tallon-Baudry \& Bertrand, 1999) for two frequency bands of interest: alpha $(8-12 \mathrm{~Hz})$ and beta (13-30 Hz; Crone et al., 1998; Willemse et al., 2010; Yuan et al., 2010). Trial averaging preceded time-frequency analysis to specifically capture task-locked modulations and minimize non-task-related signals (David, Kilner, \& Friston, 2006). Frequency-specific source time series for every subject were combined to create two data matrices (one per frequency band), each with columns corresponding to brain regions and rows corresponding to poststimulus time points $\times$ subjects. Singular value decomposition (SVD), of which PCA is a special case, was performed on each of the four standardized data matrices. For every component extracted, the resulting decomposition yielded (i) a spatial pattern of taskrelated variance in brain activity (i.e., a network constrained to the dominant $15 \%$ of interconnected brain regions derived from component loadings), resulting in independent sources of variance reflecting task-specific brain networks and (ii) component scores (i.e., time series) providing an estimate of the network's engagement at each poststimulus time point during the length of the trial, with component scores at each time point reflecting the influence of the spatial pattern for that given network. In other words, component score time series represent the strength of each network's signal, with higher/lower component scores depicting a stronger/weaker influence from that spatial pattern, and a component score of zero corresponding to baseline. The network-level connectivity analyses of oscillatory power described above were performed separately for each motor task (i.e., unimanual and bimanual).

Group differences in the activation level of each functional brain network at every poststimulus time point were statistically compared using repeated measures analysis of variance (ANOVA). Notably, repeated measures ANOVAs are suitable to test for group differences between time series as they do not assume non-independence among the repeated observations and allow for correlation between repeated measures within subjects. As such, the amplitude envelope of each network's associated time series was extracted using the Hilbert transform and then submitted to repeated measures ANOVA to test for group and time differences. For every unimanual network identified, the Hilbert transform values at each time point and for each subject were submitted
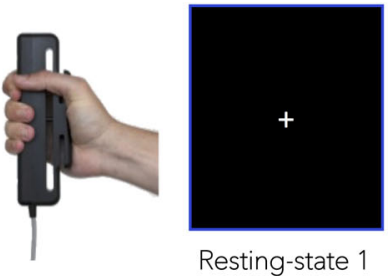

Resting-state 1

$5 \mathrm{~min}$

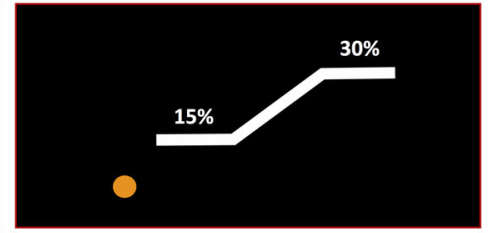

Unimanual task 50 trials | $12 \mathrm{~min}$

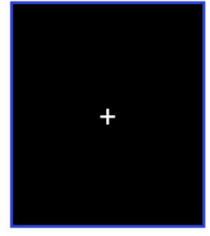

Resting-state 2

$5 \mathrm{~min}$

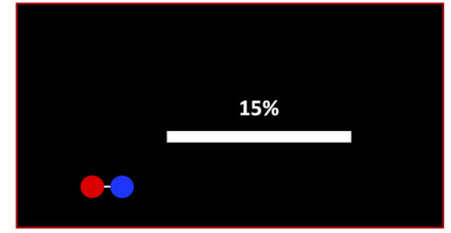

Bimanual task 50 trials $\mid \sim 10 \mathrm{~min}$

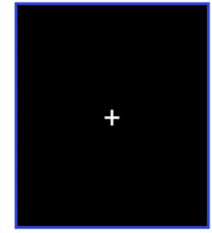

Resting-state 3 $5 \mathrm{~min}$

FIGURE 1 Schematic overview of the experiment protocol carried out in the MEG scanner. Subjects performed two hand motor control tasks (unimanual, bimanual) interspersed with three 5 min resting-state sessions. Each motor task consisted of 50 trials with variable interstimulus intervals [Color figure can be viewed at wileyonlinelibrary.com] 
to a $1,441 \times 2$ repeated-measures ANOVA, with the within-subjects factor of Poststimulus Time (1,441 time points were estimated after stimulus onset), and between-subjects factor of Group (elderly individuals and young adults). Similarly, for the identified bimanual networks, these Hilbert transform values for every subject were submitted to a $962 \times 2$ repeated-measures ANOVA, with the within-subjects factor of Poststimulus Time (962 time points), and between-subjects factor of Group. Moreover, we carried out a power analysis using G*Power (Franz Faul, Universität Kiel, Germany) and calculated the required effect sizes for the current study, with a sample size of $23\left(n_{1}=12, n_{2}=11\right)$, power $=0.8$, and alpha $=0.05$. Minimum effect sizes based on our sample size are Cohen's $f=0.046$ (unimanual task) and Cohen's $f=0.051$ (bimanual task).

\subsection{Granger causality analysis of task-specific brain networks}

Granger causality has been increasingly used to identify the presence of directional interactions (or causal relations) in physiological systems (Schiatti, Nollo, Rossato, \& Faes, 2015). This approach relies on the concept that a causal influence from a source region to a target region can be assumed if past information about the source region (i.e., its corresponding time series) improves the prediction of future values of the target region. In other words, Granger causality can provide insights as to how information propagates from one brain region to another. We conducted Granger causality connectivity analysis using the narrow band time series from each region within the task-specific, PCA-derived functional brain networks. Specifically, Granger causality was performed on each subject individually, and binary outcomes were coded 0 for nonsignificant causal relations $(p>0.05)$ and 1 for significant causal relations $(p<0.05)$ among all pairs of brain regions. The model order parameter of our multivariate autoregressive model was optimized using the minimum description length criterion and statistical significance of every pairwise causal relation was detected using an F-test. Findings were corrected for multiple comparisons, controlling at a false discovery rate of $p<0.05$. Significant group-level causality maps (constrained to the significant, subject-level causal relations) were then detected using binomial $p$ value computation for testing proportions. Specifically, for a given causal link, the binomial test uses the mean of all coded binary outcomes within a group (i.e., $0 \mathrm{~s}$ and $1 \mathrm{~s}$ ) to compute the number of subjects presenting this significant causal link that is required for this link to be significant at the grouplevel (Siegal, 1956). As such, the resulting task-based Granger causality maps display the dominant patterns of cortical information flow that were significant both at the subject- and group-levels for every task-specific brain network.

\section{5 | Resting-state functional connectivity and statistical analyses}

For each participant and each of the three resting-state sessions, the MEG data were down-sampled to $300 \mathrm{~Hz}$ and epoched offline in $10 \mathrm{~s}$ windows to obtain meaningful activation from both low- and highfrequency modulations (Supporting Information Figure S2). The choice of time window was further determined from previous work showing fairly robust test-retest reliability when using epochs of $10 \mathrm{~s}$ restingstate data (Colclough et al., 2016; Jin, Seol, Kim, \& Chung, 2011). Epochs in which significant signal artifacts were observed were rejected and the remaining "clean" $10 \mathrm{~s}$ windows were concatenated across time. The LCMV beamformer spatial filtering approach (Van Veen \& Buckley, 1988) was subsequently used on the subject-specific, concatenated data to reconstruct a single time series for all of the 148 cortical brain regions defined by the Destrieux atlas (Destrieux et al., 2010). Each time series was corrected for signal leakage effects (i.e., spurious correlations between the inferred cortical sources) using a symmetric, multivariate correction method intended for all-to-all functional connectivity analysis (Colclough, Brookes, Smith, \& Woolrich, 2015). The Hilbert transform was subsequently used to extract the instantaneous power and phase within six frequency bands of interest: delta $(1-4 \mathrm{~Hz})$, theta $(5-7 \mathrm{~Hz})$, alpha (8-12 Hz), beta (13-30 Hz), gamma "low" (31-80 Hz), and gamma "high" (81-150 Hz). Resting-state functional connectivity analysis was performed for each of the six frequency bands by systematically computing pairwise envelope correlations between all 148 source-reconstructed brain regions. The resulting all-to-all connectivity matrices (one per frequency band) were sorted in functional networks according to the recently proposed seven-network brain cortical parcellation estimated by intrinsic functional connectivity using resting-state fMRI data from 1,000 healthy adults (Yeo et al., 2011). This network parcellation provided spatial consistency across all subjects as well as between restingstate runs, thereby making direct comparison of functional network connectivity possible. Here, functional connectivity was defined as the mean connectivity strength (i.e., the mean of all pairwise correlations) within each of the pre-defined seven resting-state networks. Differences between groups (young vs. elderly), runs (resting-state 1, 2, and 3), and mean connectivity strength for each of the seven resting-state networks were identified by carrying out six $7 \times 3 \times 2$ mixed-model ANOVAs (one per frequency band). Tests of sphericity were carried out for all ANOVAs and Greenhouse-Geisser adjusted degrees of freedom were checked. Original degrees of freedom are reported as any violations of sphericity did not affect the results.

\subsection{Associations between task-based findings and resting-state connectivity changes}

To relate resting-state connectivity changes to task-based functional network activity and motor task performance, we calculated Pearson correlations between resting-state connectivity changes following both unimanual and the bimanual tasks (i.e., taken as the difference in connectivity between the second and first resting-state scan as well as between the third and second resting-state scan, respectively) and (i) the levels of coordinated activity in each task-related network and (ii) motor task performance scores.

\section{3 | RESULTS}

\section{1 | Behavioral results}

As displayed in Table 1, the behavioral scores for each hand were entered into two-sample $t$ tests to compare motor performance 
between young and elderly individuals. For both hands, young adults performed significantly better than elderly individuals on the BBT and NHPT ( $p$ values $<0.01$ ), whereas grip strength did not differ between groups ( $p$ values $>0.09$ )

As for task performance, we defined task accuracy as the difference between the grip force applied and the position of the ramp target; higher accuracy was achieved when participants closely matched the target force, defined by the middle of the target ramp. We computed the mean accuracy of all trials for every participant (within each task independently), and subsequently tested for significant differences between young and elderly individuals for each task by entering the accuracy values into two-sample $t$ tests. No significant differences were observed between both groups with respect to task accuracy during the unimanual task (using the dominant right hand; $p>0.3$ ) as well as during the bimanual task (using the dominant right hand and the non-dominant left hand; $p$ values $>0.1$ )

\section{2 | Task-based functional connectivity results}

Inspection of the scree plot of singular values was carried out for the two frequency bands of interest (alpha, beta) and for each motor task (unimanual, bimanual). Visual inspection of every component (i.e., network) extracted from our task-based analysis was performed. Brain networks which did not include motor regions were excluded from the analysis and are not discussed below. This led to the inclusion of three unimanual networks (all beta-related) and three bimanual networks (two beta-related, one alpha-related). The brain regions and estimated time series associated with each network are displayed in Figure 2a-c (unimanual networks) and Figure $3 a-c$ (bimanual networks), and described below. Anatomical descriptions for each network are presented in Supporting Information Tables S1-S3 (unimanual networks) and Supporting Information Tables S4-S6 (bimanual networks).

\section{3 | Functional networks underlying unimanual handgrips}

\subsection{1 | Ventral Frontoparietal network}

This network was the first component extracted from beta frequency activity and accounted for $18.3 \%$ of task-related variance (Supporting Information Figure S3a). Activation in this network was largely lateralized to the right hemisphere and specifically included the temporoparietal, anterior cingulate, occipital, as well as the bilateral anterior inferior frontal cortices (Figure 2a). This spatial pattern is highly consistent with that of a frontoparietal attention network known to play a role in detecting behaviorally relevant stimuli and mediating bottom-up processing (Corbetta \& Shulman, 2002; Wen, Yao, Liu, \& Ding, 2012). This network was therefore identified as the ventral frontoparietal network. The Hilbert transform values (i.e., envelope) of the network's associated time series were entered into a mixed-model ANOVA, and a significant main effect of Poststimulus Time was observed $\left(F_{1,440,30,240}=1.16, p<0.001\right)$. The Poststimulus Time $\times$ Group interaction was also significant $\left(F_{1,440}\right.$, $30,240=1.19, p<0.001 ;$ Cohen's $f=0.24)$, and was caused by increased activity in the elderly group during the sustained handgrip periods (i.e., 0-3 s and 6-9 s; Figure 2a).

\subsection{2 | Default-mode network}

This network was the second component extracted from beta frequency activity and accounted for $8.4 \%$ of task-related variance (Supporting Information Figure S3a). As displayed in Figure 2b, this network recruited core regions of the default-mode network such as the right anterior cingulate cortex, precuneus, inferior temporal cortex, as well as the bilateral ventromedial prefrontal and lateral parietal cortices. Although activation of the default-mode network has been predominantly observed under task-free or "resting-state" conditions, recent studies have reported significant default-mode network suppression during attention-demanding tasks (Anticevic et al., 2012). While a known limitation of functional connectivity measured using in vivo neuroimaging lies in an inability to distinguish excitatory from inhibitory connections (Park \& Friston, 2013), beta-related default-mode activity has been previously identified as a signature of task-related fMRI deactivations (Laufs et al., 2003). The time series envelope values associated with this network were entered into a mixed-model ANOVA; main effects of Poststimulus Time and Group, as well as the interaction, were all not significant ( $p$ values $>0.2$ ), suggesting that activity within this network did not differ across time or between groups (Figure $2 b$ ).

\subsection{3 | Motor network}

This network was the third component extracted from beta frequency activity and accounted for 5.1\% of task-related variance (Supporting Information Figure S3a). This network included predominantly left motor-related regions, notably M1, primary and secondary sensory cortices (S1, S2), and the superior frontal gyrus extending into the supplementary motor area (SMA). It also included activation in right S1 as well as the superior and inferior parietal lobules. Based on this spatial pattern (Figure 2c), this network was labeled the motor network. We found a significant main effect of Group $\left(F_{1,30,240}=4.12, p=0.05\right)$ and a significant Poststimulus Time $\times$ Group interaction $\left(F_{1,440,30,240}=1.26, p<0.001\right.$; Cohen's $f=0.25$ ). Figure $2 c$ shows that both groups produced similar levels of activity; however, relative to the young group, the time series of elderly individuals were characterized by distinct sharp activation peaks, which may underlie the significant interaction.

\subsection{Functional networks underlying bimanual handgrips}

\subsection{1 | Left-dominant motor network}

This network was the first component extracted from beta frequency activity and accounted for $19.4 \%$ of task-related variance (Supporting Information Figure S3b). Activations in this network were mostly lateralized to the left hemisphere, and included M1 extending anteriorly into PMd and PMv, S1, inferior parietal lobule, and bilateral occipital cortex (Figure 3a). This component was therefore labeled the leftdominant motor network. As evidenced by a significant Poststimulus Time $\times$ Group interaction ( $F_{961,} 22,125=1.10, p<0.05$; Cohen's $f=0.23$ ), elderly individuals exhibited distinctly higher levels of activity throughout the entire isometric bimanual handgrip relative to young adults. 
(a) VENTRAL FRONTOPARIETAL NETWORK

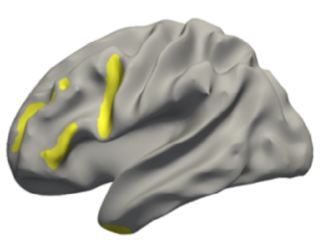

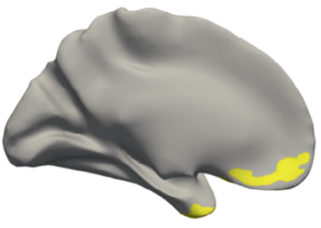

Left

(contralatera)
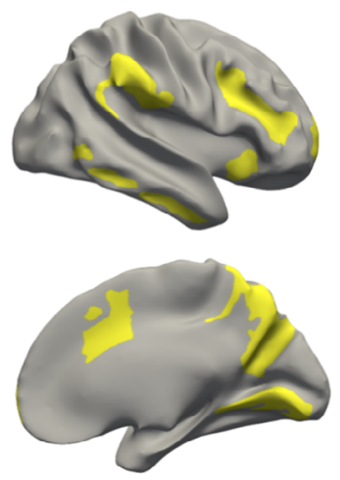

Right

(ipsilateral)

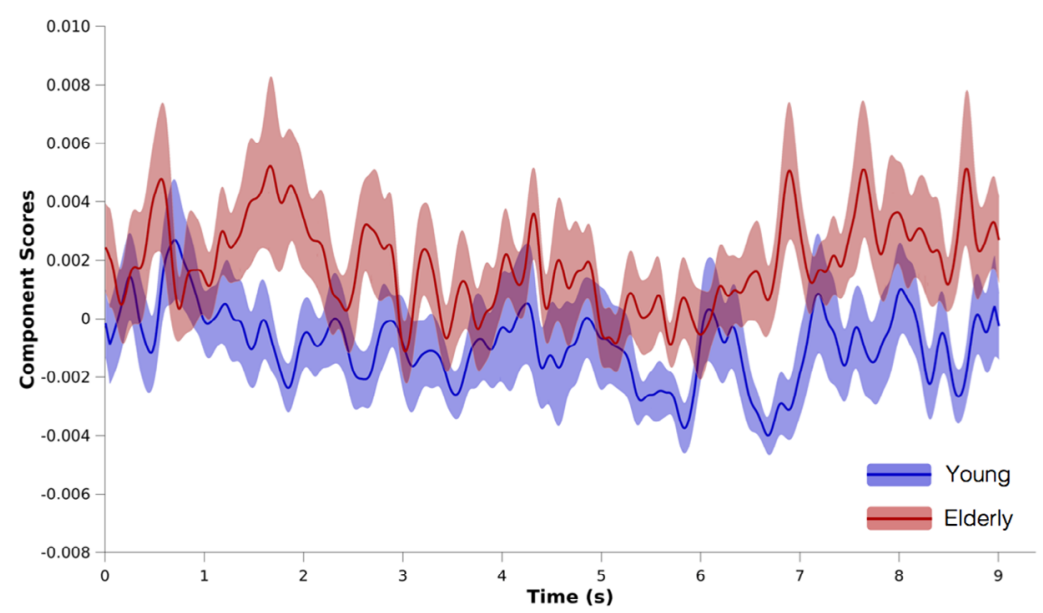

(b) DEFAULT-MODE NETWORK

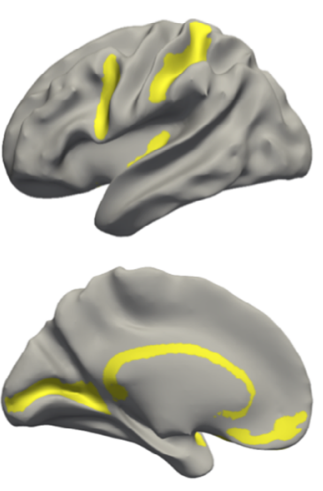

Left

(contralatera)
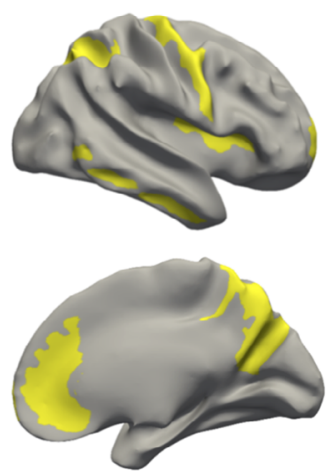

Right (ipsilatera)

(c) MOTOR NETWORK
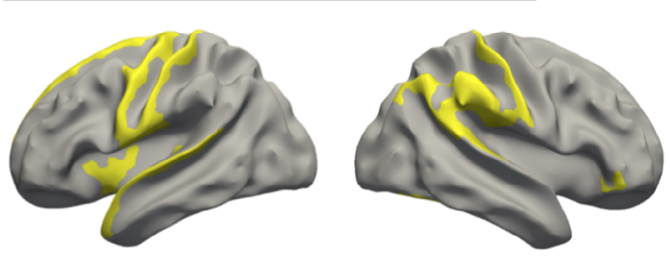

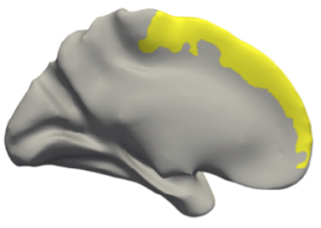

Left

(contralatera)

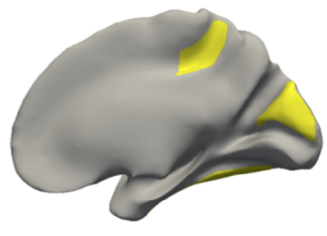

Right

(ipsilatera)
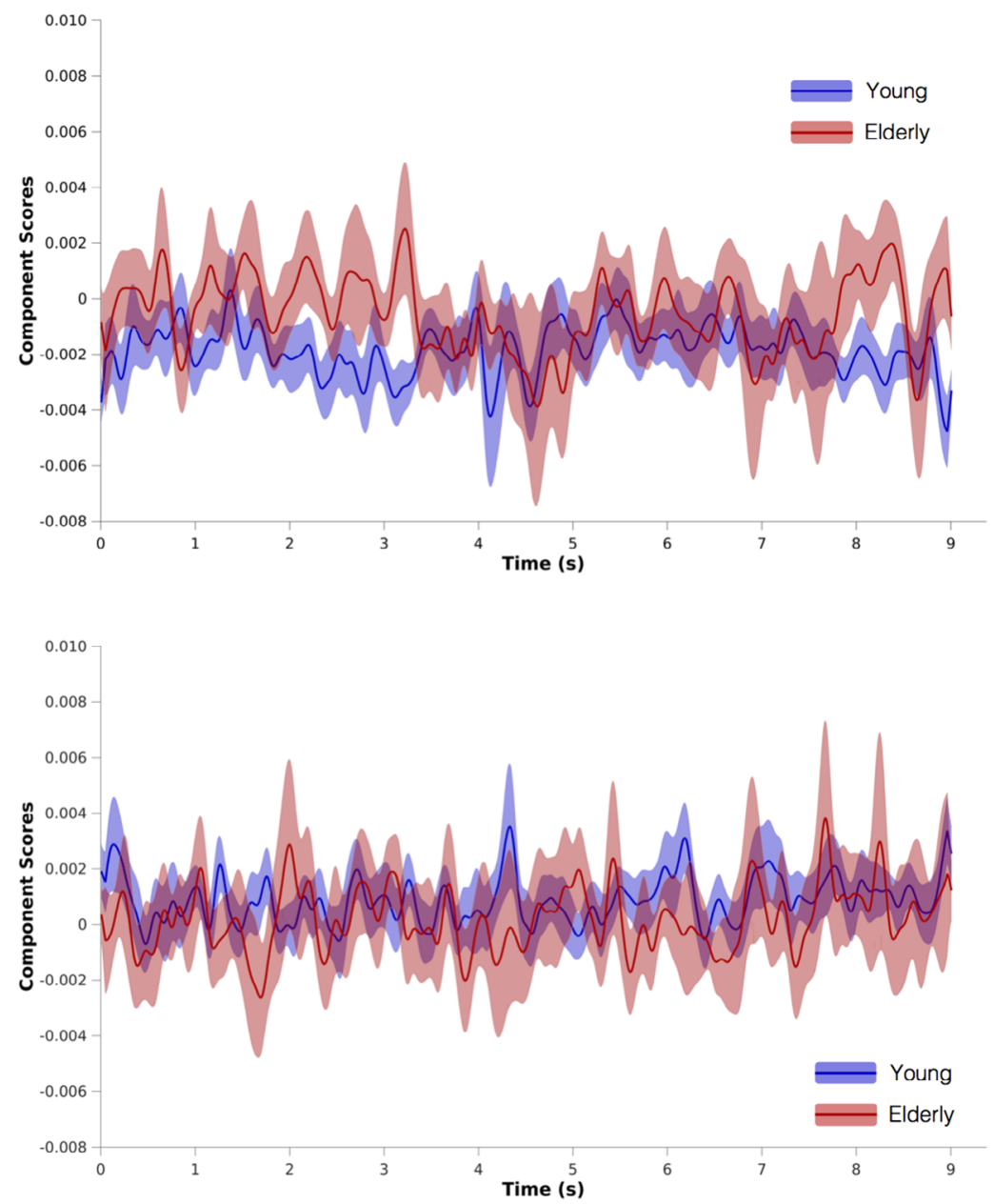

FIGURE 2 Unimanual task-based networks. (a) Ventral frontoparietal network, (b) default-mode network, (c) motor network. Compared to young adults, elderly individuals showed increased task-based connectivity in networks subserving attention-driven and motor processes. The dominant $15 \%$ of component loadings (i.e., spatial pattern) for the first three principal components extracted from the beta frequency (13-30 $\mathrm{Hz}$ ) are mapped to the surface template (left). The estimated time series (i.e., component scores) associated with each network represent the network's engagement at each poststimulus time point, with higher component scores reflecting a stronger oscillatory signal from the corresponding functionally connected network (right). The shaded area represents the standard error of the group mean. Contralateral and ipsilateral with respect to the moving (right) hand [Color figure can be viewed at wileyonlinelibrary.com]

\subsection{2 | Bilateral motor network}

This network was the second component extracted from beta frequency activity and accounted for $7.8 \%$ of task-related variance (Supporting Information Figure S3b). The spatial pattern of interconnected brain regions in this network was characterized by bilateral M1 activations (extending anteriorly into PMd), as well as left $\mathrm{S} 1$ and anterolateral parietal cortex activations (Figure 3b). This component was thus identified as the bilateral motor network. A mixed-model ANOVA carried out on the 
(a) LEFT-DOMINANT MOTOR NETWORK

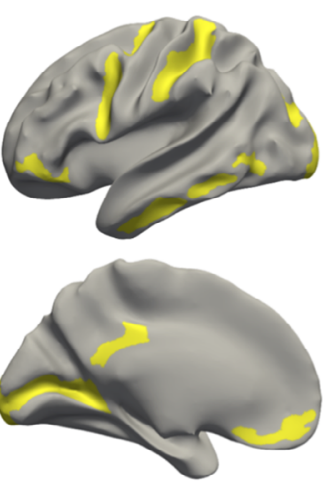

Left

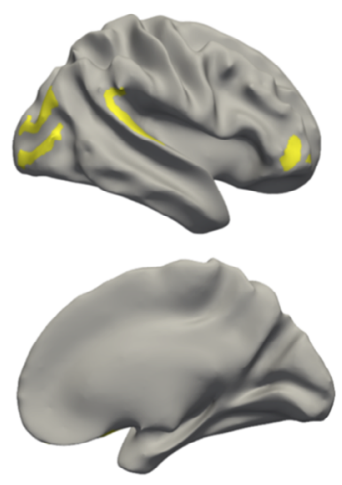

Right

(b) BILATERAL MOTOR NETWORK

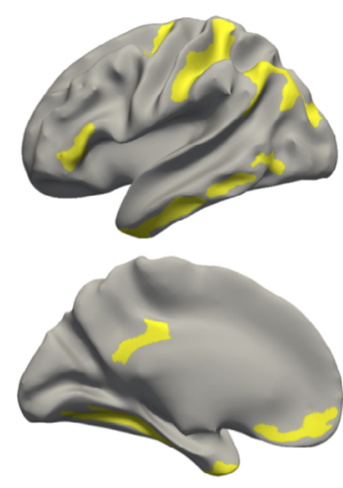

Left

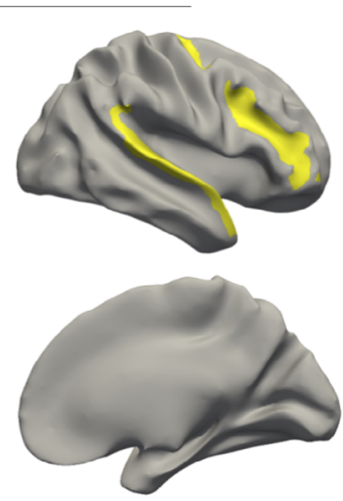

Right

(c) RIGHT-DOMINANT MOTOR NETWORK

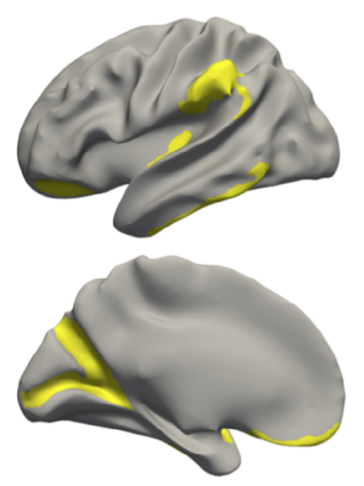

Left

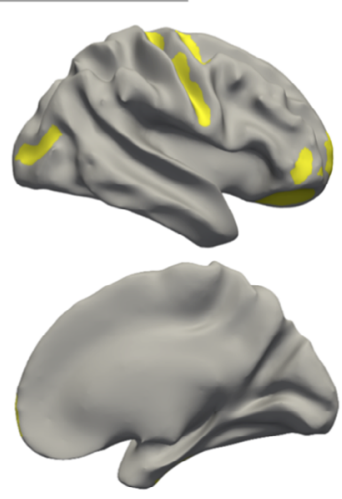

Right
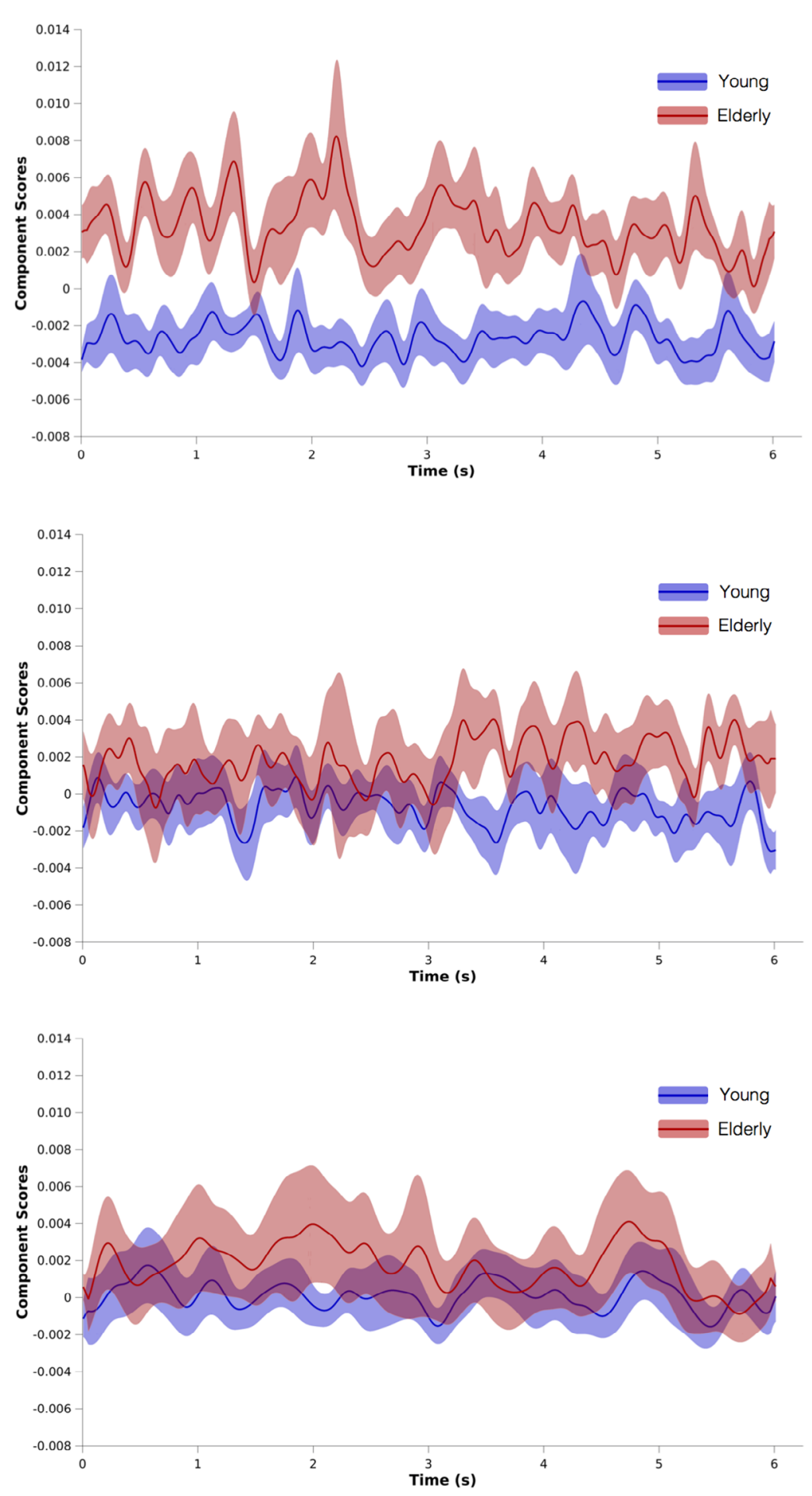

FIGURE 3 Bimanual task-based networks. (a) Left-dominant motor network, (b) bilateral motor network, (c) right-dominant motor network. Similar to the unimanual findings, relative to young adults, elderly individuals expressed varying degree of hyperconnectivity in cortical networks underlying bimanual handgrips. The dominant $15 \%$ of component loadings (i.e., spatial pattern) for the first two principal components (a and $b$ ) extracted from the beta frequency $(13-30 \mathrm{~Hz})$, and the first component (c) extracted from the alpha frequency (8-12 $\mathrm{Hz})$ are mapped to the surface template (left). The estimated time series (i.e., component scores) associated with each network represent the network's engagement at each poststimulus time point, with higher component scores reflecting a stronger oscillatory signal from the corresponding functionally connected network (right). The shaded area represents the standard error of the group mean [Color figure can be viewed at wileyonlinelibrary.com]

Hilbert transform values of the network's associated time series revealed significant main effects of Poststimulus Time $\left(F_{961}, 20,181=1.16\right.$, $p<0.0005)$, Group $\left(F_{1,20,181}=7.17, p<0.05\right)$ as well as a significant Poststimulus Time $\times$ Group interaction $\left(F_{961,22,125}=1.15, p<0.005\right.$;
Cohen's $f=0.24)$. As seen in Figure $3 b$, this interaction was caused by increased activity levels in the elderly group later in the trial (from 3 to $6 \mathrm{~s}$ ), whereas young adults exhibited constant levels of activity throughout the entire bimanual handgrip task. 


\subsection{3 | Right-dominant motor network}

This network was the first component extracted from alpha frequency activity and accounted for $25.6 \%$ of task-related variance (Supporting Information Figure S3b). The corresponding functional network included activations in and around right M1, specifically extending anteriorly into PMd and posteriorly into the central sulcus, left inferior parietal cortex, and bilateral occipital cortex (Figure 3c). Based on the spatial distribution of the network, this component was labeled as right-dominant motor network. The main effects of Poststimulus Time and Group, as well as the interaction, were not significant ( $p$ values $>0.4$ ), suggesting that activity within this network did not differ across time or between groups (Figure $3 c$ ).

\subsection{Association between behavioral scores and brain activity}

To examine the relationship between behavioral motor performance and levels of task-related brain activity, we carried out Pearson correlation analyses between task accuracy scores and the level of engagement for each functional network (i.e., the component scores). Despite the low variability in task accuracy scores between participants, older adults demonstrated a positive relationship between taskrelated brain activity and behavioral motor scores in four functional networks: default-mode and motor networks (unimanual task; Supporting Information Figure S4a), as well as left- and right-dominant motor networks (bimanual task; Supporting Information Figure S4b).

\subsection{Granger causality}

We investigated the direction of information flow from and to every brain region derived from all six task-based networks extracted from the functional connectivity analysis. Binomial statistics revealed that causal links were significant at the group-level if the links were significant at the individual-level in at least five subjects (i.e., 5/12 for the young group and 5/11 for the elderly group), that is: $p$ value $=\mathrm{P}\left(X \geq 5 \mid p=\mu_{\text {group }}\right)<0.05$. We used a multivariate autoregressive model of order 3 , meaning that the time lag between interacting neuronal ensembles corresponded to 18.75 ms (i.e., 3/160). Granger causality maps for the unimanual and bimanual networks as well as for each group are depicted in Figure 4a-f, respectively. Brain region abbreviations for the Granger causality maps are listed in Table 2.

\section{7 | Resting-state functional connectivity results}

A significant main effect of Run was observed in two frequency bands, specifically delta $\left(F_{2,42}=11.1, p<0.005\right)$ and beta $\left(F_{2,42}=4.7\right.$, $p<0.05$ ), whereas a significant main effect of Network was found in all frequency bands: delta $\left(F_{6,126}=14.3, p<0.001\right)$, theta $\left(F_{6,126}=14.3\right.$, $p<0.001)$, alpha $\left(F_{6,126}=56.3, p<0.001\right)$, beta $\left(F_{6}, 126=14.3\right.$, $p<0.001)$, gamma "low" ( $\left.F_{6,126}=8.8, p<0.001\right)$, and gamma "high" $\left(F_{6,126}=8.0, p<0.001\right)$. Significant interactions involving Network Run, or Group were solely observed in the delta and beta frequency bands and are described below.

\subsection{Task-induced connectivity changes in the delta frequency band}

Slow oscillatory connectivity (1-4 Hz) differed between young and elderly subjects across different resting-state sessions as evidenced by a significant Run $\times$ Group interaction $\left(F_{2,42}=5.61, p<0.05, \eta_{\mathrm{p}}^{2}=0.21\right)$. Within-subjects contrasts yielded significant group differences from the first to second resting-state run $(p<0.005)$, and from the second to the third run $(p<0.05)$. As can be seen from Figure $5 \mathrm{a}$, this interaction was caused by elderly subjects exhibiting a large increase in delta connectivity in the second resting-state run (i.e., after the unimanual task) relative to young adults. Correlations between unimanual task-based functional findings and resting-state connectivity changes (i.e., difference between second and first resting-state scans) revealed a significant relationship between ventral frontoparietal network activity and resting-state delta increases following the unimanual task in older adults $(r=0.53, p<0.05)$. Moreover, a negative association between delta-related resting-state connectivity changes and task performance was observed in young adults $(r=-0.50, p<0.05)$ but was marginal in elderly individuals $(r=-0.30$, $p>0.18$; Supporting Information Figure S5a). The analogous correlations involving resting-state connectivity changes following the bimanual task were all nonsignificant (Supporting Information Figure S6a).

\section{9 | Task-induced connectivity changes in the beta frequency band}

The beta frequency $(13-30 \mathrm{~Hz})$ showed a significant Run $\times$ Network interaction $\left(F_{12,252}=2.76, p<0.05, \eta_{p}^{2}=0.12\right)$, indicating that beta-related resting-state network connectivity varies as a function of time (i.e., resting-state run). Figure $5 \mathrm{~b}$ shows that this interaction can be interpreted by enhanced connectivity from the first to the second resting run (i.e., increased connectivity after the unimanual task), notably in the visual, dorsal attention, and sensorimotor networks. A significant Network $x$ Group interaction was also observed $\left(F_{6,126}=3.43, p<0.05, \eta_{p}^{2}=0.14\right)$ suggesting that elderly subjects demonstrated slightly higher beta oscillatory connectivity than young adults in all resting-state networks (nonsignificant, $p$ values $>0.36$ ), with the exception of the visual network $(p<0.05$; Figure 5c). Correlations between unimanual and bimanual taskbased functional findings and resting-state connectivity changes did not yield any significant associations; however, older adults demonstrated a strong positive relationship between beta-related resting-state connectivity increases following unimanual handgrips and task performance $(r=0.64, p<0.05)$, an association that was absent in young individuals $(r=0.20, p>0.26$; Supporting Information Figures S5b,S6b).

\section{4 | DISCUSSION}

We compared functional brain network organization in young and elderly individuals during and before/after unimanual and bimanual hand movements. Capitalizing on the high spatiotemporal resolution of MEG, we employed an innovative combination of functional and effective multivariate connectivity analyses to derive task-specific brain networks and assess the direction of information flow among cortical areas. Relative to young adults, elderly individuals showed hyperactivity in networks 
Unimanual Task

(a)
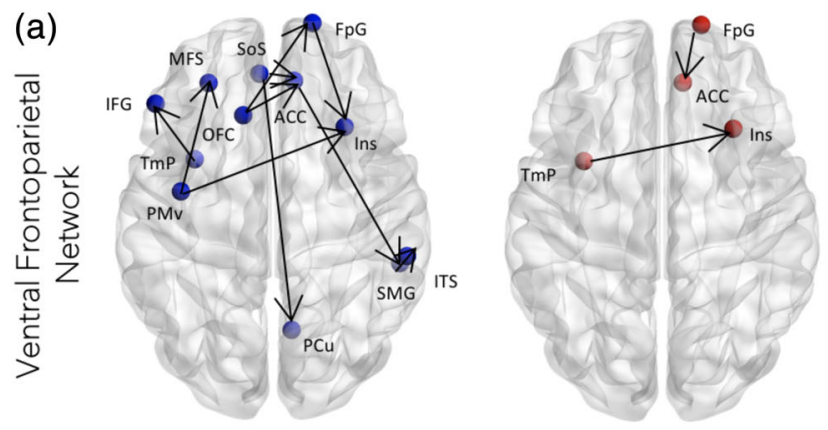

(b)

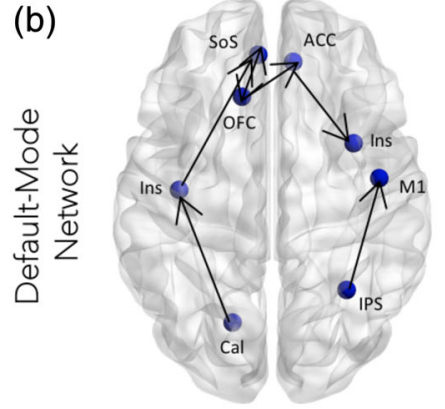

(c)
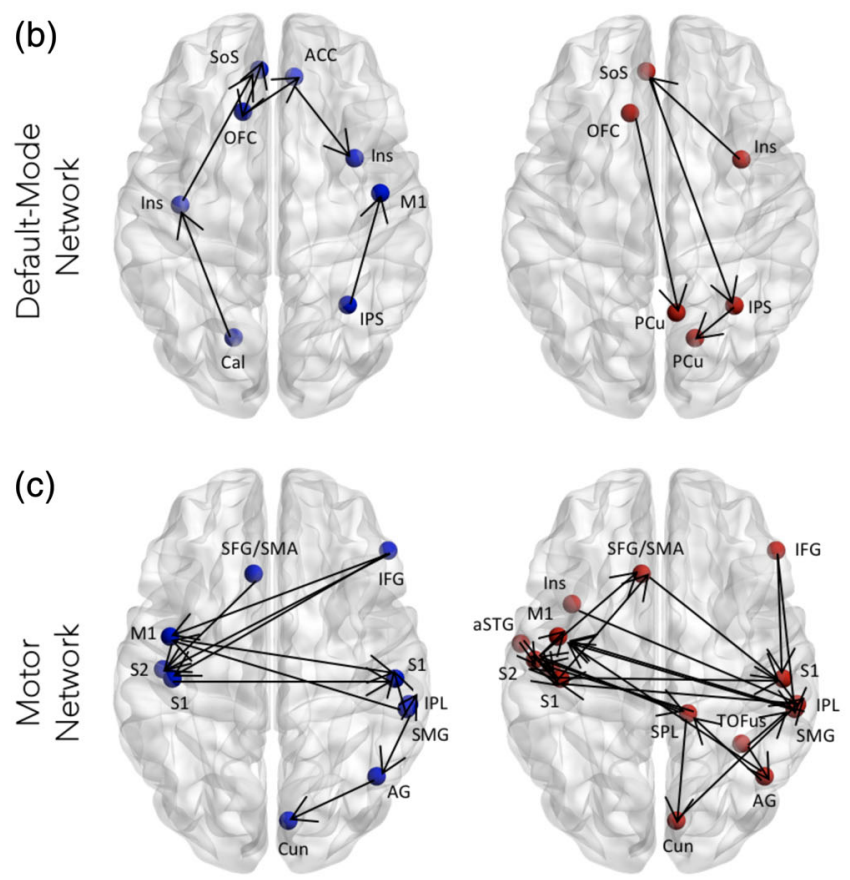

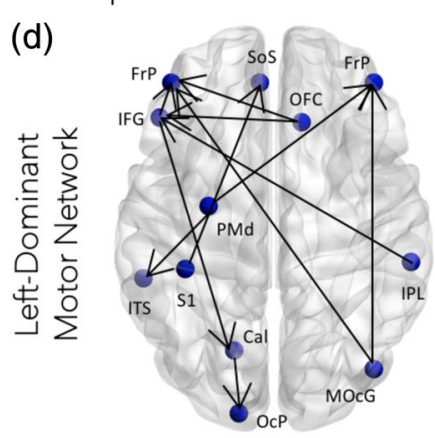

Bimanual Task
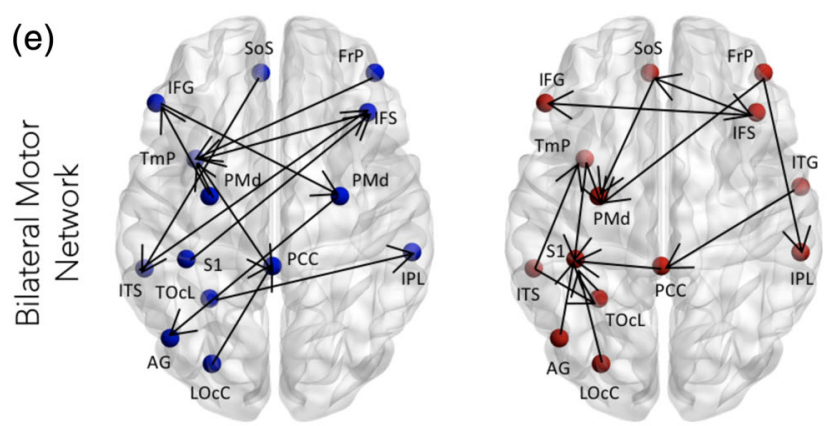

(f)
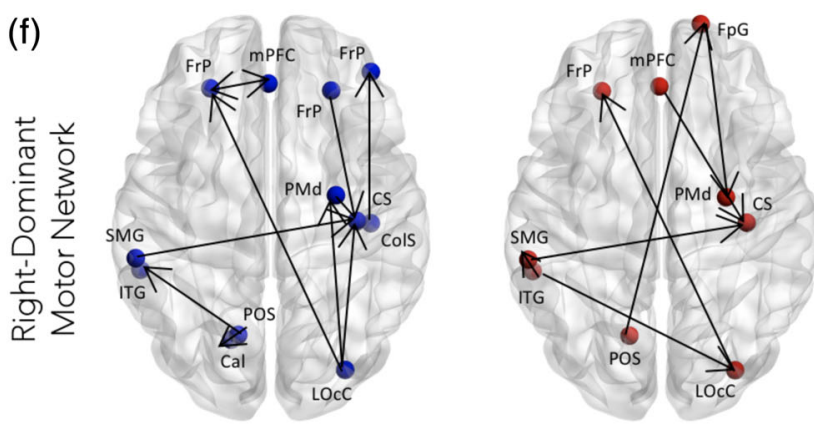

- Young

- Elderly

FIGURE 4 Within-network effective connectivity. Group-specific granger causality maps for the unimanual and bimanual task-based brain networks are displayed in $(a-c)$ and $(d-f)$, respectively. Relative to young adults, elderly individuals displayed greater information flow from contralateral parietal and ipsilateral secondary motor regions to the left primary motor cortex during the unimanual task as well as decreased interhemispheric temporo-frontal communication during the bimanual task. Surface brains with blue nodes represent causal maps for the young group and those with red nodes represent causal maps for the elderly group. Abbreviations for each brain region are listed in Table 2 [Color figure can be viewed at wileyonlinelibrary.com]

subserving attention-driven and motor processes, in both unimanual and bimanual hand motor control tasks. Granger causality analysis of taskbased networks revealed that elderly individuals were characterized by an increased input to $\mathrm{M} 1$ contralateral to the hand used during the unimanual task, whereas during the bimanual task, they exhibited a relative decrease in frontal connectivity. Taken together, these findings highlight a novel age-related dichotomy in hemispheric communication during unimanual and bimanual movements, with increased ipsilateral to contralateral communication during the unimanual task and a substantial decrease in interhemispheric communication during the bimanual task. Furthermore, by measuring resting-state activity levels before and after each motor task, we found that elderly individuals, similarly to young adults, maintain the capacity to adapt to task demands via network-wide connectivity increases. Collectively, our multi-method approach quantifies functional and effective macroscale cortical organization changes, and further expands our understanding of neuronal flexibility following motor processes during aging.

\subsection{Age-related connectivity changes in unimanual movements}

During movement and performance of visuomotor control tasks, primary and secondary sensorimotor networks are engaged to implement motor behavior, while networks comprising higher-order regions are recruited to ensure cognitive resources and goal-directed behavior (Serrien, Ivry, \& Swinnen, 2007). Indeed, tasks that demand externalized attention to a 
TABLE 2 Granger causality brain region abbreviations

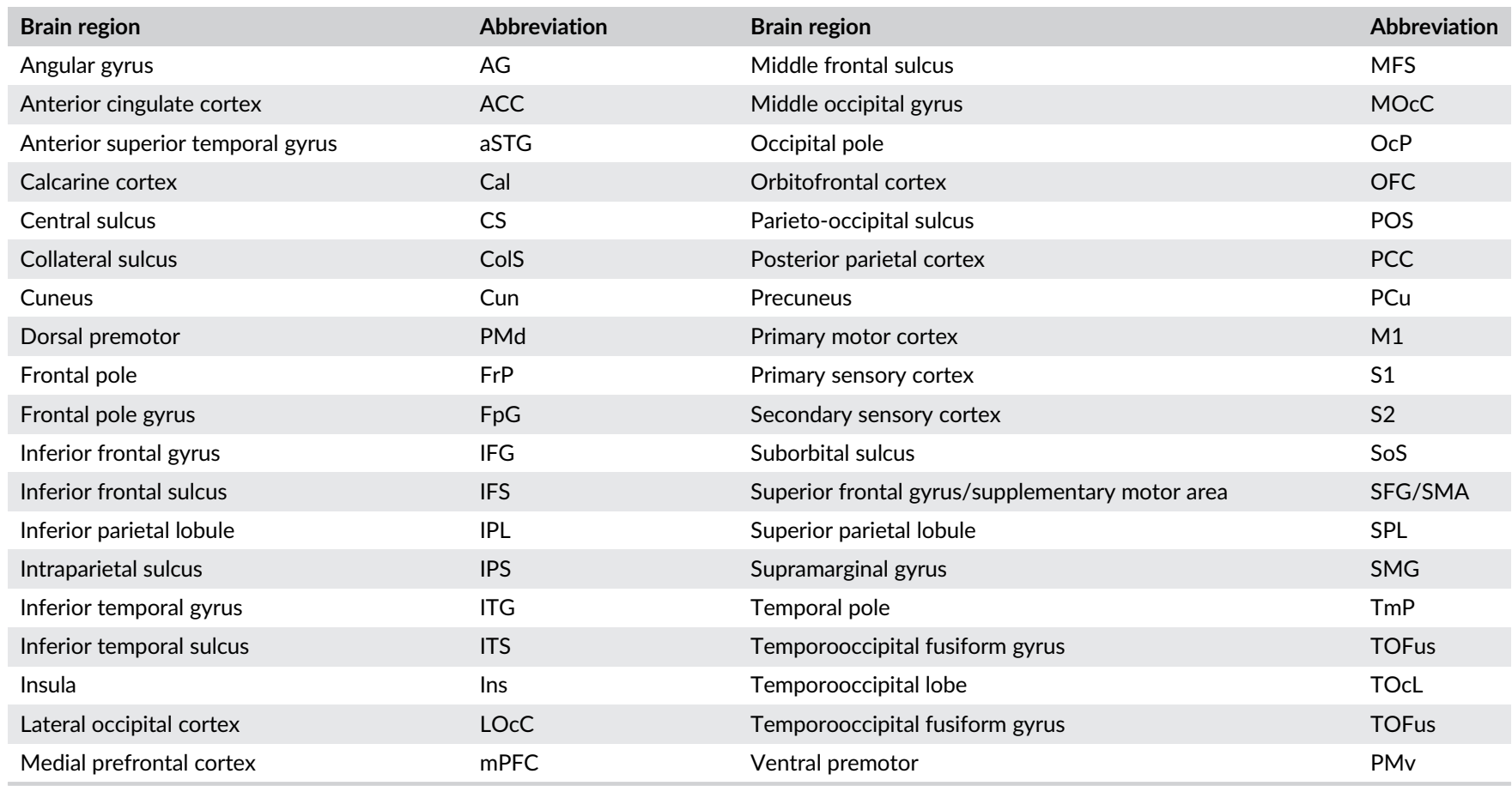

visually presented stimulus have been shown to reliably activate regions within the frontoparietal network (Corbetta \& Shulman, 2002; Spreng, Stevens, Chamberlain, Gilmore, \& Schacter, 2010), with older adults showing higher activity increases in the prefrontal and parietal cortex (Cabeza et al., 2002; Madden et al., 2007). Our findings revealed a similar pattern of hyperconnectivity during the unimanual task, suggesting that attentional demands are significantly increased when elderly participants are required to perform handgrips. Accordingly, this greater cortical activation could further reflect a mechanism by which goal-directed attentional control is employed by elderly individuals in an attempt to dampen the processing of task-irrelevant stimuli (Milham et al., 2002). Unlike the attention network, in which increased activation was indicated by overall higher oscillatory amplitude, enhanced activity in the motor network was characterized by sharp beta peaks in elderly individuals. According to computational modeling studies on transient neocortical beta rhythms, these sharp beta oscillations likely reflect greater temporal synchrony in synaptic input on cortical pyramidal neurons (Sherman et al., 2016). In line with the latter finding, our effective connectivity analysis based on Granger causality revealed enhanced interhemispheric information flow from ipsilateral (right) primary and secondary sensorimotor regions as well as parietal regions to contralateral (left) M1 during unimanual handgrips in the elderly group. Combined, these findings are in agreement with an influential theory of brain reorganization suggesting a loss of asymmetry in connectivity patterns in older adults (Heuninckx et al., 2005, 2008; Ward \& Frackowiak, 2003), possibly reflecting a mechanism whereby greater synchrony of excitatory input currents are recruited to (a) GROUP x RUN | DELTA (1-4 HZ)

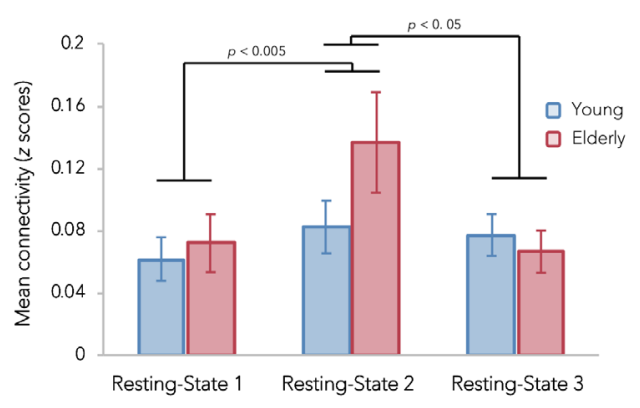

(b) RUN x NETWORK | BETA (13-30 Hz)

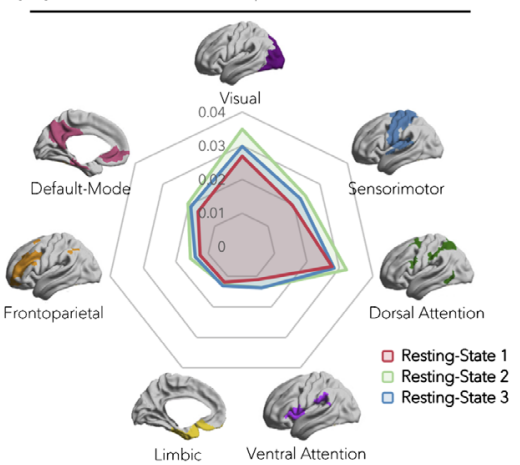

(c) GROUP $x$ NETWORK | BETA (13-30 HZ)

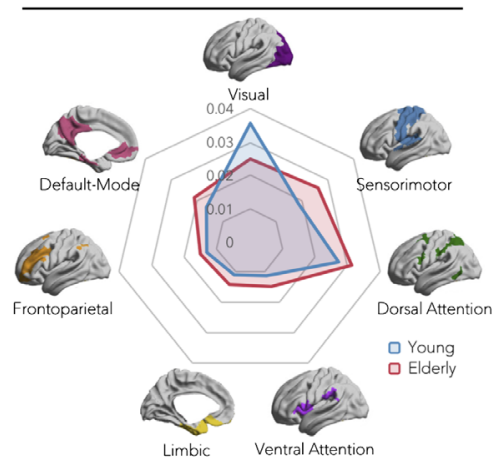

FIGURE 5 Task-induced resting-state connectivity modulation. (a) Group difference in mean resting-state connectivity in the delta frequency range (1-4 Hz), averaged across all networks, and plotted as a function of resting-state run. (b) Spider plot showing mean beta-related (13-30 $\mathrm{Hz}$ ) connectivity differences between resting-state runs, averaged across groups, and plotted as a function of resting-state networks; relative to baseline (Resting-State 1), all seven resting-state networks showed task-induced connectivity increases. (c) Spider plot showing group differences in mean beta-related connectivity, averaged across resting-state runs, and plotted as a function of resting-state networks; relative to young adults, elderly individuals showed higher connectivity levels in all but the visual network [Color figure can be viewed at wileyonlinelibrary.com] 
counteract known age-related structural (Salat et al., 2004, 2005), myeloarchitectural (Bartzokis, 2004), and neurochemical changes (Mora et al., 2008). Future neuroimaging studies integrating network connectivity measures with microstructural features, such as myelination and tissue microstructure, may enrich our understanding of the intriguing associations existing between beta oscillatory rhythms and myelin in aging populations as well as in neurodegenerative disorders (Lariviere et al., 2018).

\subsection{Age-related connectivity changes in bimanual movements}

Neurophysiological and neuroimaging studies of bimanual hand movements support the view that the dominant hemisphere, as opposed to each contralateral hemisphere independently, controls the organization of bimanual hand movements (Serrien, Cassidy, \& Brown, 2003). In line with these earlier observations, our whole-brain multivariate analysis of the bimanual task data revealed three functional networks of interest: a left-dominant, a right-dominant, and a bilateral motor network. Akin to our unimanual findings, we found age-related connectivity increases in the left-dominant and bilateral motor networks during sustained isometric bimanual handgrips. Interestingly, while the unimanual task only elicited networks in the beta band, both alpha and beta motor networks emerged during the bimanual task. Based on previous electrophysiological and fMRI studies highlighting the importance of the dominant hemisphere during bimanual hand movements (Jancke et al., 1998; Serrien et al., 2003; Viviani, Perani, Grassi, Bettinardi, \& Fazio, 1998), we speculate that task-related activity from the nondominant side (i.e., right hemisphere) is suppressed-or driven into a more "rest-like" state-by the dominant hemisphere, an interaction that may be accompanied by more pronounced alpha brain waves. Alternatively, it has been suggested that beta-related activity preferentially relates to motor aspects of coordination while alpharelated activity underlies somatosensory processing (Pfurtscheller, Stancak Jr., \& Neuper, 1996; Salmelin \& Hari, 1994). In line with our current findings, this alpha-beta dichotomy may reflect different systems whereby beta-related dominant (i.e., left) and bilateral motor network activity is involved in the initial production of bimanual handgrips, whereas alpha-related nondominant (i.e., right) motor network activity rather reflects a mechanism by which the nondominant hemisphere processes sensory information from the dominant hemisphere and adjust its motor output accordingly.

The effective connectivity maps for the bimanual task-based networks revealed that young adults rely more on prefrontal regions, such as the frontal pole, suborbital sulcus, orbitofrontal cortex, and medial prefrontal cortex. This pattern of strongly interconnected frontal regions was largely absent in the elderly group, which is in agreement with previous cognitive studies reporting age-related activity decreases in prefrontal regions (Cabeza et al., 2002; Damoiseaux et al., 2007). Yet another striking difference in connectivity patterns between young and older adults lies in the importance of the left temporal pole region in the bilateral motor network. According to our Granger causality analysis, this brain area appears highly integrated within the bilateral motor network in the young group as it receives cortical information from several parietal and frontal regions (including left PMd), but also has a causal influence on the posterior cingulate cortex, a connectivity pattern that is largely absent in elderly individuals. Our findings further demonstrate that bimanual movements in elderly individuals appear to be controlled from each contralateral hemisphere independently-with minimal interhemispheric connectivity-thus expanding upon previous research suggesting that the neural organization of the bilateral motor network in adults is driven by the dominant (left) hemisphere (Serrien et al., 2003). This lack of coordination between hemispheres may result from altered white matter integrity of the corpus callosum, which plays a key role in allowing both hemispheres to communicate during bimanual movements (Kennerley, Diedrichsen, Hazeltine, Semjen, \& Ivry, 2002; Ota et al., 2006). Indeed, previous research investigating the interhemispheric connections of the temporal lobes in monkeys demonstrated that the corpus callosum receives extensive fibers from the temporal pole (Demeter, Rosene, \& Van Hoesen, 1990). This reinforces the importance of the left temporal pole in coordinating movement in both hands during bimanual movements. Our results further suggest that this region is a central hub responsible for mediating information flow between the two hemispheres. Due to their long-distance connectivity and their central role in communication and integration of information in the brain, cortical hubs are known to be represent a high-cost feature of brain connectivity and as a consequence become highly vulnerable in aging (Crossley et al., 2014).

\section{3 | Task-induced modulation of resting-state network connectivity}

Previous research studying the effects of intensive motor learning on subsequent resting-state brain activity in healthy adults have confirmed the presence of task-induced short- and long-term changes in cortical properties (Bellec et al., 2015; Vahdat, Darainy, Milner, \& Ostry, 2011). In line with these reports, our analysis of the resting-state runs acquired before and after each motor task revealed significant connectivity increases following the unimanual task relative to baseline (i.e., the first resting-state session), but significant connectivity decreases following the bimanual task (relative to the second resting-state session). This enhanced connectivity pattern was observed in both young and elderly subjects, and was particularly noticeable in resting-state cortical networks that included regions previously engaged during the unimanual task (e.g., visual, dorsal attention, sensorimotor, default-mode networks). On the other hand, the lack of task-induced connectivity increases in the third resting-state scan (i.e., following the bimanual task) may relate to longer trial duration in the unimanual task ( $9 \mathrm{~s} \mathrm{unimanual} \mathrm{handgrips} \mathrm{vs.} 6 \mathrm{~s}$ bimanual handgrips) which in turn yielded a stronger and/or longerlasting postmovement rebound effect. Notably, our data suggest that unimanual hand movements can induce similar neuronal flexibility, as reflected by an increase in resting brain connectivity relative to baseline, even in the absence of external stimulation or intensive motor learning. However, future research protocols encompassing an aspect of motor learning, whereby motor performance changes across time, may be better suited to study the aging brain's ability to adapt to task demands. While the evidence reported herein indicates that healthy adults, irrespective of age, retain the capacity for task-induced connectivity changes at a systems level, elderly individuals also showed task-induced 
increases in slow wave oscillatory connectivity. Considered alongside cognitive studies in aging which have reported enhanced interhemispheric delta coherence (Maurits, Scheeringa, van der Hoeven, \& de Jong, 2006; Vecchio, Miraglia, Bramanti, \& Rossini, 2014), the large increase in delta connectivity following the unimanual task may play a role in modulating attentional or cognitive resources, and may therefore represent a marker of healthy neurocognitive aging.

\section{4 | Limitations, methodological considerations, and future directions}

We investigated group differences in activity levels of commonly shared task-based brain networks which precluded us from determining whether, and how, spatial reorganization occurs during healthy aging. While these questions were outside the scope of this study, our Granger causal connectivity approach provided initial insights into age-related changes in the spatial pattern of information flow involved in motor processes. Further, the relatively small number of participants in each group may have contributed to low statistical power and an inability to detect significant group differences. While results reported here are consistent with previous $\mathrm{fMRI}$ and EEG studies with larger sample sizes (Maes et al., 2017; Sala-Llonch, Bartrés-Faz, \& Junqué, 2015), reproducibility of our findings should be assessed in a similar, but larger, cohort of young and elderly individuals. Lastly, our choice of processing pipeline may have precluded the identification of modulatory effects that were not timelocked to the production of isometric handgrips. While our trial averaged connectivity analyses may have led to nonphase-locked task-related signal loss in alpha and beta frequency bands (Brookes et al., 2016), our analytical framework was primarily designed to target oscillations that were directly evoked by the production of isometric handgrips while also suppressing non-task-related signals as well as physiological and measurement noise (David et al., 2006).

\section{5 | Conclusions}

The present work shows that despite matching levels of task accuracy, brain organization in elderly individuals is characterized by hyperactivity in MEG networks specifically underlying the performance of unimanual and bimanual hand movements. Furthermore, spatial patterns of information flow involved in motor processes significantly differed between young and older adults, with the latter group showing enhanced connectivity from ipsilateral frontal and parietal motor regions to contralateral M1 during unimanual movements. Interestingly, while frontal and temporal regions acted as integrative hubs for the coordination of interhemispheric information flow in young adults, elderly individuals demonstrated relatively little fronto-temporal information flow during the bimanual task. Finally, the combination of task-related and restingstate protocols described here represents a valuable tool for future research aiming at understanding brain organization during healthy aging. Our integrative experimental approach may further contribute to the refinement of rehabilitation strategies aiming at enhancing neural flexibility in movement-impaired populations by means of exercise programs targeting hand movement.

\section{ACKNOWLEDGMENTS}

The authors would like to thank Elizabeth Bock for assistance with MEG data acquisition as well as Arna Gosh for his support in implementing the visual feedback for the experiment. S.L. acknowledges funding from Fonds de la Recherche du Québec--Santé (FRQS). A.X.P. and M.K. received financial support through graduate scholarships from McGill University. G.N. receives financial support from an AXA Research Fund postdoctoral fellowship. G.D.M. and M.-H.B. are supported by Fonds de la Recherche du Québec--Nature et Techonologies (FRQNT; 2016-PR191780) and the Canadian Foundation from Innovation (M.-H. B.: 34277, G.D.M.: 34362). S.B. is supported by a Discovery Grant from the Natural Science and Engineering Research Council of Canada (NSERC; 436355-13), the National Institutes of Health $(\mathrm{NIH}$; 2R01EB009048-05) and a Platform Support Grant from the Brain Canada Foundation (PSG15-3755).

\section{CONFLICT OF INTEREST}

The authors declare no conflict of interest.

\section{ORCID}

Sara Larivière (D) https://orcid.org/0000-0001-5701-1307

\section{REFERENCES}

Anticevic, A., Cole, M. W., Murray, J. D., Corlett, P. R., Wang, X.-J., \& Krystal, J. H. (2012). The role of default network deactivation in cognition and disease. Trends in Cognitive Sciences, 16, 584-592.

Baillet, S. (2017). Magnetoencephalography for brain electrophysiology and imaging. Nature Neuroscience, 20, 327-339.

Bartzokis, G. (2004). Age-related myelin breakdown: A developmental model of cognitive decline and Alzheimer's disease. Neurobiology of Aging, 25, 5-18.

Bellec, P., Benhajali, Y., Carbonell, F., Dansereau, C., Albouy, G., Pelland, M., ... Stip, E. (2015). Impact of the resolution of brain parcels on connectomewide association studies in $\mathrm{fMRI}$. Neurolmage, 123, 212-228.

Brookes, M. J., Tewarie, P. K., Hunt, B. A., Robson, S. E., Gascoyne, L. E., Liddle, E. B., ... Morris, P. G. (2016). A multi-layer network approach to MEG connectivity analysis. Neurolmage, 132, 425-438.

Bruce, E. N., Bruce, M. C., \& Vennelaganti, S. (2009). Sample entropy tracks changes in EEG power spectrum with sleep state and aging. Journal of Clinical Neurophysiology: Official Publication of the American Electroencephalographic Society, 26, 257-266.

Cabeza, R., Anderson, N. D., Locantore, J. K., \& Mclntosh, A. R. (2002). Aging gracefully: Compensatory brain activity in high-performing older adults. Neurolmage, 17, 1394-1402.

Calautti, C., Serrati, C., \& Baron, J. C. (2001). Effects of age on brain activation during auditory-cued thumb-to-index opposition: A positron emission tomography study. Stroke, 32, 139-146.

Colclough, G., Brookes, M. J., Smith, S. M., \& Woolrich, M. W. (2015). A symmetric multivariate leakage correction for MEG connectomes. Neurolmage, 117, 439-448.

Colclough, G. L., Woolrich, M. W., Tewarie, P., Brookes, M. J., Quinn, A. J., \& Smith, S. M. (2016). How reliable are MEG resting-state connectivity metrics? Neurolmage, 138, 284-293.

Corbetta, M., \& Shulman, G. L. (2002). Control of goal-directed and stimulus-driven attention in the brain. Nature Reviews Neuroscience, 3 , 201-215.

Crone, N. E., Miglioretti, D. L., Gordon, B., Sieracki, J. M., Wilson, M. T., Uematsu, S., \& Lesser, R. P. (1998). Functional mapping of human sensorimotor cortex with electrocorticographic spectral analysis I. Alpha and beta event-related desynchronization. Brain, 121(Pt 12), 2271-2299. 
Crossley, N. A., Mechelli, A., Scott, J., Carletti, F., Fox, P. T., McGuire, P., \& Bullmore, E. T. (2014). The hubs of the human connectome are generally implicated in the anatomy of brain disorders. Brain, 137, 2382-2395.

Damoiseaux, J., Beckmann, C., Arigita, E. S., Barkhof, F., Scheltens, P., Stam, C., ... Rombouts, S. (2007). Reduced resting-state brain activity in the "default network" in normal aging. Cerebral Cortex, 18, 1856-1864.

David, O., Kilner, J. M., \& Friston, K. J. (2006). Mechanisms of evoked and induced responses in MEG/EEG. Neurolmage, 31, 1580-1591.

Demeter, S., Rosene, D. L., \& Van Hoesen, G. W. (1990). Fields of origin and pathways of the interhemispheric commissures in the temporal lobe of macaques. Journal of Comparative Neurology, 302, 29-53.

Destrieux, C., Fischl, B., Dale, A., \& Halgren, E. (2010). Automatic parcellation of human cortical gyri and sulci using standard anatomical nomenclature. Neurolmage, 53, 1-15.

Enoka, R. M., Christou, E. A., Hunter, S. K., Kornatz, K. W., Semmler, J. G., Taylor, A. M., \& Tracy, B. L. (2003). Mechanisms that contribute to differences in motor performance between young and old adults. Journal of Electromyography and Kinesiology, 13, 1-12.

Fujiyama, H., Van Soom, J., Rens, G., Gooijers, J., Leunissen, I., Levin, O., \& Swinnen, S. P. (2016). Age-related changes in frontal network structural and functional connectivity in relation to bimanual movement control. The Journal of Neuroscience, 36, 1808-1822.

Heuninckx, S., Wenderoth, N., Debaere, F., Peeters, R., \& Swinnen, S. P. (2005). Neural basis of aging: The penetration of cognition into action control. Journal of Neuroscience, 25, 6787-6796.

Heuninckx, S., Wenderoth, N., \& Swinnen, S. P. (2008). Systems neuroplasticity in the aging brain: Recruiting additional neural resources for successful motor performance in elderly persons. Journal of Neuroscience, 28, 91-99.

Hong, S. L., \& Rebec, G. V. (2012). A new perspective on behavioral inconsistency and neural noise in aging: Compensatory speeding of neural communication. Frontiers in Aging Neuroscience, 4, 27.

Jancke, L., Peters, M., Schlaug, G., Posse, S., Steinmetz, H., \& MullerGartner, H. (1998). Differential magnetic resonance signal change in human sensorimotor cortex to finger movements of different rate of the dominant and subdominant hand. Brain Research. Cognitive Brain Research, 6, 279-284.

Jin, S. H., Seol, J., Kim, J. S., \& Chung, C. K. (2011). How reliable are the functional connectivity networks of MEG in resting states? Journal of Neurophysiology, 106, 2888-2895.

Kennerley, S. W., Diedrichsen, J., Hazeltine, E., Semjen, A., \& Ivry, R. B. (2002). Callosotomy patients exhibit temporal uncoupling during continuous bimanual movements. Nature Neuroscience, 5, 376-381.

Kielar, A., Deschamps, T., Chu, R. K., Jokel, R., Khatamian, Y. B., Chen, J. J., \& Meltzer, J. A. (2016). Identifying dysfunctional cortex: Dissociable effects of stroke and aging on resting state dynamics in MEG and fMRI. Frontiers in Aging Neuroscience, 8, 40. https://doi.org/ 10.3389/fnagi.2016.00040. eCollection 2016.

King, B. R., van Ruitenbeek, P., Leunissen, I., Cuypers, K., Heise, K. F., Santos Monteiro, T., ... Swinnen, S. P. (2017). Age-related declines in motor performance are associated with decreased segregation of large-scale resting state brain networks. Cereb Cortex, 28(12), 4390-4402. https://doi. org/10.1093/cercor/bhx297.

Krueger, H., Koot, J., Hall, R. E., O'callaghan, C., Bayley, M., \& Corbett, D. (2015). Prevalence of individuals experiencing the effects of stroke in Canada. Stroke, 46, 2226-2231.

Larivière, S., Lavigne, K. M., Woodward, T. S., Gerretsen, P., GraffGuerrero, A., \& Menon, M. (2017). Altered functional connectivity in brain networks underlying self-referential processing in delusions of reference in schizophrenia. Psychiatry Research: Neuroimaging, 263, 32-43.

Lariviere, S., Vos de Wael, R., Paquola, C., Hong, S. J., Misic, B., Bernasconi, N., ... Bernhardt, B. (2018). Microstructure-informed connectomics: Enriching large-scale descriptions of healthy and diseased brains. Brain Connect. https://doi.org/10.1089/brain.2018.0587. [Epub ahead of print]

Larivière, S., Ward, N. S., \& Boudrias, M. H. (2018). Disrupted functional network integrity and flexibility after stroke: Relation to motor impairments. Neurolmage: Clinical, 19, 883-891.

Laufs, H., Krakow, K., Sterzer, P., Eger, E., Beyerle, A., Salek-Haddadi, A., \& Kleinschmidt, A. (2003). Electroencephalographic signatures of attentional and cognitive default modes in spontaneous brain activity fluctuations at rest. Proceedings of the National Academy of Sciences of the United States of America, 100, 11053-11058.

Ma, L., Wang, B., Narayana, S., Hazeltine, E., Chen, X., Robin, D. A., ... Xiong, J. (2010). Changes in regional activity are accompanied with changes in inter-regional connectivity during 4 weeks motor learning. Brain Research, 1318, 64-76.

Madden, D. J., Spaniol, J., Whiting, W. L., Bucur, B., Provenzale, J. M., Cabeza, R., ... Huettel, S. A. (2007). Adult age differences in the functional neuroanatomy of visual attention: A combined $\mathrm{fMRI}$ and DTI study. Neurobiology of Aging, 28, 459-476.

Maes, C., Gooijers, J., de Xivry, J.-J. O., Swinnen, S. P., \& Boisgontier, M. P. (2017). Two hands, one brain, and aging. Neuroscience \& Biobehavioral Reviews, 75, 234-256.

Mattay, V. S., Fera, F., Tessitore, A., Hariri, A. R., Das, S., Callicott, J. H., \& Weinberger, D. R. (2002). Neurophysiological correlates of age-related changes in human motor function. Neurology, 58, 630-635.

Maurits, N. M., Scheeringa, R., van der Hoeven, J. H., \& de Jong, R. (2006). EEG coherence obtained from an auditory oddball task increases with age. Journal of Clinical Neurophysiology, 23, 395-403.

Milham, M. P., Erickson, K. I., Banich, M. T., Kramer, A. F., Webb, A., Wszalek, T., \& Cohen, N. J. (2002). Attentional control in the aging brain: Insights from an fMRI study of the stroop task. Brain and Cognition, 49, 277-296.

Mora, F., Segovia, G., \& del Arco, A. (2008). Glutamate-dopamine-GABA interactions in the aging basal ganglia. Brain Research Reviews, 58, 340-353.

O'Neill, G. C., Tewarie, P. K., Colclough, G. L., Gascoyne, L. E., Hunt, B. A., Morris, P. G., ... Brookes, M. J. (2017). Measurement of dynamic task related functional networks using MEG. Neurolmage, 146, 667-678.

Oldfield, R. C. (1971). The assessment and analysis of handedness: The Edinburgh inventory. Neuropsychologia, 9, 97-113.

Ota, M., Obata, T., Akine, Y., Ito, H., Ikehira, H., Asada, T., \& Suhara, T. (2006). Age-related degeneration of corpus callosum measured with diffusion tensor imaging. Neurolmage, 31, 1445-1452.

Park, C.-h., Boudrias, M.-H., Rossiter, H., \& Ward, N. S. (2012). Age-related changes in the topological architecture of the brain during hand grip. Neurobiology of Aging, 33, 833.e27-833.e37.

Park, H.-J., \& Friston, K. (2013). Structural and functional brain networks: From connections to cognition. Science, 342, 1238411.

Pfurtscheller, G., Stancak, A., Jr., \& Neuper, C. (1996). Event-related synchronization (ERS) in the alpha band--An electrophysiological correlate of cortical idling: A review. International Journal of Psychophysiology, 24, 39-46.

Sala-Llonch, R., Bartrés-Faz, D., \& Junqué, C. (2015). Reorganization of brain networks in aging: A review of functional connectivity studies. Frontiers in Psychology, 6, 663.

Salat, D., Tuch, D., Greve, D., Van Der Kouwe, A., Hevelone, N., Zaleta, A., ... Rosas, H. D. (2005). Age-related alterations in white matter microstructure measured by diffusion tensor imaging. Neurobiology of Aging, 26, 1215-1227.

Salat, D. H., Buckner, R. L., Snyder, A. Z., Greve, D. N., Desikan, R. S., Busa, E., ... Fischl, B. (2004). Thinning of the cerebral cortex in aging. Cerebral Cortex, 14, 721-730.

Salmelin, R., \& Hari, R. (1994). Characterization of spontaneous MEG rhythms in healthy adults. Electroencephalography and Clinical Neurophysiology, 91, 237-248.

Schiatti, L., Nollo, G., Rossato, G., \& Faes, L. (2015). Extended granger causality: A new tool to identify the structure of physiological networks. Physiological Measurement, 36, 827-843.

Seidler, R. D., Bernard, J. A., Burutolu, T. B., Fling, B. W., Gordon, M. T., Gwin, J. T., ... Lipps, D. B. (2010). Motor control and aging: Links to age-related brain structural, functional, and biochemical effects. Neuroscience \& Biobehavioral Reviews, 34, 721-733.

Serrien, D. J., Cassidy, M. J., \& Brown, P. (2003). The importance of the dominant hemisphere in the organization of bimanual movements. Human Brain Mapping, 18, 296-305.

Serrien, D. J., Ivry, R. B., \& Swinnen, S. P. (2007). The missing link between action and cognition. Progress in Neurobiology, 82, 95-107.

Sherman, M. A., Lee, S., Law, R., Haegens, S., Thorn, C. A., Hämäläinen, M. S., ... Jones, S. R. (2016). Neural mechanisms of transient neocortical beta rhythms: Converging evidence from humans, 
computational modeling, monkeys, and mice. Proceedings of the National Academy of Sciences, 113, E4885-E4894.

Siegal, S. (1956). Nonparametric statistics for the behavioral sciences. New York, NY: McGraw-hill.

Siman-Tov, T., Bosak, N., Sprecher, E., Paz, R., Eran, A., Aharon-Peretz, J., \& Kahn, I. (2016). Early age-related functional connectivity decline in highorder cognitive networks. Frontiers in Aging Neuroscience, 8, 330.

Solesio-Jofre, E., Beets, I. A. M., Woolley, D. G., Pauwels, L., Chalavi, S., Mantini, D., \& Swinnen, S. P. (2018). Age-dependent modulations of resting state connectivity following motor practice. Frontiers in Aging Neuroscience, 10, 25.

Song, J., Birn, R. M., Boly, M., Meier, T. B., Nair, V. A., Meyerand, M. E., \& Prabhakaran, V. (2014). Age-related reorganizational changes in modularity and functional connectivity of human brain networks. Brain Connectivity, 4, 662-676.

Spreng, R. N., Stevens, W. D., Chamberlain, J. P., Gilmore, A. W., \& Schacter, D. L. (2010). Default network activity, coupled with the frontoparietal control network, supports goal-directed cognition. Neurolmage, 53, 303-317.

Sullivan, E. V., Rohlfing, T., \& Pfefferbaum, A. (2010). Quantitative fiber tracking of lateral and interhemispheric white matter systems in normal aging: Relations to timed performance. Neurobiology of Aging, 31, 464-481.

Supek, S., \& Aine, C. J. (2014). Magnetoencephalography: From signals to dynamic cortical networks. Heidelberg, Germany: Springer.

Tadel, F., Baillet, S., Mosher, J. C., Pantazis, D., \& Leahy, R. M. (2011). Brainstorm: A user-friendly application for MEG/EEG analysis. Computational Intelligence and Neuroscience, 2011, 879716.

Tallon-Baudry, C., \& Bertrand, O. (1999). Oscillatory gamma activity in humans and its role in object representation. Trends in Cognitive Sciences, 3, 151-162.

Tomasi, D., \& Volkow, N. D. (2012). Aging and functional brain networks. Molecular Psychiatry, 17, 549-558.

Tung, K. C., Uh, J., Mao, D., Xu, F., Xiao, G., \& Lu, H. (2013). Alterations in resting functional connectivity due to recent motor task. Neurolmage, 78, 316-324.

Vahdat, S., Darainy, M., Milner, T. E., \& Ostry, D. J. (2011). Functionally specific changes in resting-state sensorimotor networks after motor learning. Journal of Neuroscience, 31, 16907-16915.

Van Veen, B. D., \& Buckley, K. M. (1988). Beamforming: A versatile approach to spatial filtering. IEEE ASSP Magazine, 5, 4-24.

Vecchio, F., Miraglia, F., Bramanti, P., \& Rossini, P. M. (2014). Human brain networks in physiological aging: A graph theoretical analysis of cortical connectivity from EEG data. Journal of Alzheimer's Disease, 41, 1239-1249.
Viviani, P., Perani, D., Grassi, F., Bettinardi, V., \& Fazio, F. (1998). Hemispheric asymmetries and bimanual asynchrony in left- and right-handers. Experimental Brain Research, 120, 531-536.

Vlahou, E. L., Thurm, F., Kolassa, I.-T., \& Schlee, W. (2014). Resting-state slow wave power, healthy aging and cognitive performance. Scientific Reports, 4, 5101.

Ward, N., \& Frackowiak, R. (2003). Age-related changes in the neural correlates of motor performance. Brain, 126, 873-888.

Ward, N. S., Swayne, O. B., \& Newton, J. M. (2008). Age-dependent changes in the neural correlates of force modulation: An fMRI study. Neurobiology of Aging, 29, 1434-1446.

Wen, X., Yao, L., Liu, Y., \& Ding, M. (2012). Causal interactions in attention networks predict behavioral performance. Journal of Neuroscience, 32, 1284-1292.

Whitman, J. C., Takane, Y., Cheung, T. P. L., Moiseev, A., Ribary, U., Ward, L. M., \& Woodward, T. S. (2016). Acceptance of evidencesupported hypotheses generates a stronger signal from an underlying functionally-connected network. Neurolmage, 127, 215-226.

Willemse, R. B., de Munck, J. C., Verbunt, J. P., van 't Ent, D., Ris, P., Baayen, J. C., ... Vandertop, W. P. (2010). Topographical organization of $\mathrm{mu}$ and Beta band activity associated with hand and foot movements in patients with perirolandic lesions. Open Neuroimaging Journal, 4, 93-99.

Yeo, B. T., Krienen, F. M., Sepulcre, J., Sabuncu, M. R., Lashkari, D., Hollinshead, M., ... Polimeni, J. R. (2011). The organization of the human cerebral cortex estimated by intrinsic functional connectivity. Journal of Neurophysiology, 106, 1125-1165.

Yuan, H., Liu, T., Szarkowski, R., Rios, C., Ashe, J., \& He, B. (2010). Negative covariation between task-related responses in alpha/beta-band activity and BOLD in human sensorimotor cortex: An EEG and fMRI study of motor imagery and movements. Neurolmage, 49, 2596-2606.

\section{SUPPORTING INFORMATION}

Additional supporting information may be found online in the Supporting Information section at the end of this article.

How to cite this article: Larivière $S$, Xifra-Porxas $A$, Kassinopoulos $\mathrm{M}$, et al. Functional and effective reorganization of the aging brain during unimanual and bimanual hand movements. Hum Brain Mapp. 2019;40:3027-3040. https://doi.org/ 10.1002/hbm.24578 